\title{
Compestoto
}

\section{As publicações dos pesquisadores brasileiros da área da Saúde: a questão do acesso}

\author{
Luiza Helena Goulart da Silva \\ Mestre; Universidade Federal de Santa Catarina (UFSC), Florianópolis, SC, Brasil; \\ luizagsilva@gmail.com \\ Rosângela Schwarz Rodrigues \\ Doutora; Universidade Federal de Santa Catarina (UFSC), Florianópolis, SC, Brasil; \\ rosangela@cin.ufsc.br \\ Gleisy Regina Bories Fachin \\ Doutora; Universidade Federal de Santa Catarina (UFSC), Florianópolis, SC, Brasil; \\ gleisy.fachin@ufsc.br
}

\begin{abstract}
Resumo: Esta pesquisa analisou a produção científica de pesquisadores brasileiros na área da Saúde. A metodologia é exploratória, descritiva e documental com avaliação predominantemente quantitativa. O corpus da pesquisa é composto por artigos publicados pelos pesquisadores, com bolsa de produtividade do CNPq, nos anos de 2010, 2011 e 2012. A amostra da pesquisa contempla 39 pesquisadores que estão vinculados a instituições em que os repositórios estejam registrados no ROAR e OPENDOAR. Os resultados mostram que os pesquisadores possuem um grande número de artigos publicados, principalmente em periódicos americanos e ingleses. Os artigos publicados em periódicos brasileiros se encontram em acesso aberto. Do total de 1.296 artigos publicados, $12 \%$ encontram-se nos repositórios das instituições pesquisadas.
\end{abstract}

Palavras-chave: Produção científica. Comunicação científica. Pesquisadores. Periódicos científicos. Repositórios institucionais.

\section{Introdução}

A comunicação científica encontra-se no coração da ciência, constituindo-se parte essencial do processo de investigação e garantindo a vitalidade da própria ciência, envolvendo todas as atividades associadas a produção, disseminação e uso da informação (MEADOWS, 1999; GARVEY, 1979). É ela a responsável pela publicação dos avanços das pesquisas em todas as áreas do conhecimento, e sua divulgação é importante para que outros pesquisadores possam colaborar com novas pesquisas ou divergir sobre determinado assunto (LEITE, 2009).

A atividade científica que não é escrita e comunicada, de acordo com Alves (2009, p. 104), perde o sentido, já que as instituições de pesquisa e os 
pesquisadores são avaliados pelo que publicam. As atividades científicas podem ser publicadas em vários tipos de suportes e podem estar em formato de relatórios, trabalhos apresentados em congressos, palestras, livros em papel ou em meio eletrônico, ou artigos publicados em periódicos científicos.

Os periódicos científicos digitais são definidos por Gruszynski e Golin (2007) como aqueles disponíveis on-line, que adotam padrões de cientificidade e de responsabilidade de instituições afins, como universidades, sociedades e órgãos de pesquisa, independentemente de possuírem uma versão impressa.

Outra forma de disponibilizar os artigos científicos em acesso aberto são os Repositórios Digitais, que tomaram uma maior dimensão a partir do Movimento do Acesso Aberto e as Tecnologias da Informação e Comunicação (TICs). Os Repositórios Institucionais foram concebidos para manter e gerenciar documentos de instituições por um tempo indeterminado e disponibilizá-los ao público, além de dar uma maior visibilidade para a produção científica de pesquisadores e instituições (RODRIGUES, 2004; GUÉDON, 2010).

A grande ambição do acesso aberto é possibilitar a igualdade de acesso à informação entre países desenvolvidos e em desenvolvimento, contribuindo para minimizar as diferenças geográficas e financeiras dos pesquisadores localizados em regiões distantes dos centros de pesquisa mais avançados (GUÉDON, 2010).

Por esses motivos, procurou-se, neste artigo, identificar o tipo de acesso da produção científica dos pesquisadores 1A [classificação da CAPES (2014b) para pesquisadores que tem produção científica elevada e coordenadores de grupos de pesquisa consolidados e reconhecidos], da área da Saúde, em periódicos científicos e repositórios. A escolha da área da Saúde se justifica pela relevância que possui no âmbito da produção e da publicação científicas. $O$ Plano Nacional de Pós-Graduação (PNPG), 2011-2020 (CAPES, 2014a, p. 562) aponta que, no Brasil, a área da Saúde Humana é o setor de atividade de pesquisa de maior dimensão em número de linhas de pesquisa e de pesquisadores envolvidos. Esse estudo também aponta que, entre os anos de 2000 e 2008, a área da Saúde Humana foi responsável por cerca de 25\% de toda a atividade de pesquisa no país. Rodrigues e Abadal (2014) destacam que a Saúde é a área com maior número de títulos indexados nas bases de dados Web 
of Science (WoS) e SciVerse Scopus (SCOPUS) no Brasil, em 2013, responsável por $22 \%$ (54) do total de revistas.

Definiu-se como objetivos específicos: a) descrever os periódicos em que os pesquisadores $1 \mathrm{~A}$ publicaram artigos; b) quantificar o número de artigos publicados pelos pesquisadores na área da Saúde; c) identificar o tipo de acesso dos periódicos; d) apontar o acervo disponível nos repositórios institucionais, dos pesquisadores 1A/Saúde, em repositórios registrados no ROAR e OPENDOAR.

\section{Revisão de literatura}

Os periódicos científicos, de acordo com Alves (2011, p. 1), “[...] são os canais de comunicação mais utilizados, dada sua própria constituição, que tem como premissa a comunicação científica dos feitos de uma determinada época [...]”. Têm como missão, segundo Packer (2011, p. 30), “[...] a comunicação dos resultados da pesquisa original, o que contribui para o avanço do conhecimento nas disciplinas ou áreas temáticas específicas [...]”.

Os periódicos são formados por artigos com resultados de pesquisas de uma mesma área, tendo como característica o registro e a preservação do conhecimento de determinada área do conhecimento. Também servem a mais três propósitos: a) a comunicação entre cientistas; b) a divulgação de resultados de pesquisa e dos estudos acadêmicos; c) estabelecimento da prioridade científica (MUELLER, 1999).

Os artigos publicados em periódicos incluídos em bases de dados, como a WOS e SCOPUS, são considerados os mais importantes em função do rigor da seleção dos títulos nas duas bases e, como resultado, os autores tendem a referenciá-los mais em suas próprias obras. À medida que são citadas pelos autores de diferentes países, as publicações se tornam mais relevantes internacionalmente (BUELA-CASAL, ZYCH, 2012).

Sobre os periódicos eletrônicos de acesso aberto, Prosser (2004, p. 2) “[...] explica que são aqueles que não cobram pelo acesso aos documentos, mas os torna disponíveis eletronicamente para todos [...]”. Além das revistas, que são totalmente de acesso aberto e as de acesso por subscrição, existem aquelas em 
que as editoras comerciais publicam cobrando taxas dos autores. Dois desses tipos de acesso são: o aberto comercial e o por subscrição com opção autor paga.

O acesso por subscrição é oferecido por revistas que seguem o sistema tradicional de assinaturas e mantêm os direitos autorais dos trabalhos. Esse tipo de acesso acontece tanto para periódicos impressos quanto para a versão digital e pode ser considerado um entrave ao avanço do conhecimento global, pois somente universidades e pesquisadores mais ricos têm acesso à maioria das revistas (SCIENTIFIC JOURNALS INTERNATIONAL, 2012, p. 1).

O acesso por subscrição com opção autor paga aparece em periódicos em que o acesso pode ser aberto ou por subscrição. $\mathrm{O}$ acesso aos artigos depende de assinaturas, mas é permitido aos autores colocar seu artigo disponível para todos pagando uma taxa (GUANAES, GUIMARÃES, 2012; FREIRE, 2011; MELERO; ABAD GARCIA, 2011; MIGUEL, CHINCHILLA-RODRÍGUEZ; MOYA-ANEGÓN, 2011; GUMIEIRO, 2009).

A primeira editora que adotou o acesso por subscrição com opção autor paga foi a SPRINGER, com a criação do Springer Open Choice, lançado em 2010 (MELERO; ABAD GARCIA, 2011; FREIRE, 2011; GUÉDON, 2010). No acesso aberto não comercial, as despesas de publicação são assumidas pelas instituições, financiamentos governamentais ou agência de fomento (GUANAES; GUIMARÃES, 2012; FREIRE, 2011; MELERO; ABAD GARCIA, 2011; GUMIEIRO, 2009; COSTA, 2006).

Segundo Rodrigues e Abadal, (2014, p. 2146) no Brasil, mais de 95\% dos periódicos de qualidade são publicados em acesso aberto, incluindo aqueles indexados em bases de prestígio como WOS e SCOPUS. Estudos da Comissão Europeia destacam a liderança do Brasil em proporção de artigos disponíveis em acesso aberto. Esse modo de publicação foi viabilizado principalmente no Brasil e nos demais países da América Latina graças a dois motivos principais: a) primeiro, o fato de a maioria dos periódicos serem editados por sociedades e associações científicas ou instituições universitárias sem fins lucrativos, que fazem uso das contribuições dos seus associados e de subsídio governamental para custear as publicações; b) segundo, o surgimento da plataforma SCIELO, em 1998 (SCIELO em Perspectiva, 2013). 
O modelo de acesso aberto comercial é também conhecido como "taxa de processamento de artigos" ou Article Processing Fees (APC). Nesse modelo, o acesso é livre ao leitor e os custos são repassados aos autores (GUANAES; GUIMARÃES, 2012).

Umas das primeiras editoras a usar o sistema autor paga foi a BIOMED Central, fundada em 1999. A Public Library of Science (PLoS One) é outro exemplo de editora comercial que utiliza esse sistema. Atualmente, ela publica oito revistas em biomedicina e é basicamente financiada com contribuições dos autores, mas também tem um sistema de financiamento institucional, membros individuais, patrocinadores e doações (MELERO; ABAD GARCIA, 2011).

Esses novos modelos de negócios, junto com as TICs e as alternativas de acesso aberto, fizeram com que outras maneiras de divulgação de pesquisas surgissem, entre elas, a inclusão dos Repositórios Institucionais, que se tornou um novo modelo de acesso aberto, também chamado de Via Verde.

As primeiras iniciativas de instalação dos repositórios digitais datam da década de 1990 e o primeiro repositório a ser implementado foi o de Los Alamos National Laboratory Phisys Archive, atual ArXiv, fundado em 1991 por Paul Ginsparg (TOMAÉL; SILVA, 2007).

Segundo Abadal (2012, p. 45), os repositórios institucionais são aqueles que "[...] contêm a produção de membros de uma instituição, que pode ser uma universidade ou um centro de investigação [...]". Assim, vários países criaram repositórios, mas ainda há relativamente poucos depósitos nos mesmos, segundo Harnad (2007), isso está relacionado à não obrigatoriedade do depósito nos repositórios institucionais.

No Brasil, a disponibilização de resultados de pesquisas em canais de comunicação de acesso aberto, principalmente em repositórios, não é ainda uma realidade para todas as áreas do conhecimento (CHALHUB, 2010).

Os periódicos científicos em acesso aberto e os repositórios contribuem para que muitas das barreiras impostas pelas editoras comerciais sejam eliminadas, permitindo que muitas das pesquisas relevantes sejam divulgadas e acessadas por pesquisadores de todos os países. 


\section{Metodologia}

A pesquisa é de caráter exploratório e descritivo, classificada como bibliográfica e documental e, quanto à forma de abordagem, caracteriza-se como quantitativa.

O universo da pesquisa foram os artigos científicos publicados nos anos de 2010, 2011 e 2012 pelos pesquisadores da área da Saúde classificados como 1A pelo Conselho Nacional de Desenvolvimento Cientifico e Tecnológico (CNPq). Segundo o CNPq (2013), os pesquisadores 1A são aqueles que têm mostrado excelência continuada na produção científica e na formação de recursos humanos e que lideram grupos de pesquisa consolidados. A escolha da utilização do triênio (2010-2012) se deu porque se refere a um triênio completo de avaliação da CAPES.

Uma vez identificados os pesquisadores da área da Saúde, tabulou-se os dados dos mesmos, selecionando os que apresentavam vínculo com instituições que possuíam repositórios registrados nos diretórios Registry of Open Access Repositories (ROAR) ou Directory of Open Access Repositories (OPENDOAR). Na categoria institucional, o Brasil possuía 69 repositórios registrados no ROAR e 54 no OPENDOAR, totalizando 123. Adotaram-se dois critérios para a seleção da amostra: a instituição possuir repositório registrado nos dois diretórios citados e ter pesquisador classificado como 1A pelo CNPq na área da Saúde.

A coleta de dados terminou em dezembro de 2013, quando foi realizada uma revisão em todos os dados pesquisados. $\mathrm{Na}$ data de início da pesquisa, o CNPq possuía 15.108 pesquisadores divididos nas mais diversas áreas e nas seguintes classificações: 1A, 1B, 1C, 1D e 2. Destes, 1.260 são classificados como 1A, distribuídos em 90 instituições.

Para a identificação dos pesquisadores 1A da área da Saúde, foi utilizado o site do CNPq, no qual se coletou o nome do pesquisador e as instituições às quais estão vinculados. A partir do Currículo Lattes de cada pesquisador, utilizando a ferramenta indicadores de produção, foram identificados os periódicos nos quais os artigos foram publicados. Então, foram coletadas as informações do número de artigos produzidos nos anos de 2010, 2011 e 2012 e os periódicos em que foram publicados. 
A identificação do país de cada periódico (distribuição geográfica) foi realizada no site do próprio periódico, assim como o tipo de entidade editora e o tipo de acesso aos periódicos e artigos. A identificação dos endereços eletrônicos (URL) e a data de criação dos repositórios foram realizadas por meio dos dois diretórios Registry of Open Access Repositories (ROAR) ou Directory of Open Access Repositories (OPENDOAR). Nos sites dos repositórios, foram identificados o número de artigos publicados, os títulos dos periódicos e o tipo de acesso aos trabalhos.

\section{Resultados e discussão}

$\mathrm{Na}$ primeira análise, foram identificados 131 pesquisadores, divididos em 23 instituições. Como nem todas as instituições possuíam repositórios registrados no ROAR ou OPENDOAR (delimitação da pesquisa) foram selecionadas 12 instituições e 39 pesquisadores, resultando no corpus da pesquisa.

A grande área da saúde, segundo a classificação da CAPES, divide-se em: Medicina, Nutrição, Odontologia, Farmácia, Enfermagem, Saúde Coletiva e Educação Física. Sendo que, nessa pesquisa, Nutrição e Educação Física não apresentam pesquisador $1 \mathrm{~A}$ vinculado à instituição que tivesse repositório registrado.

A organização por subárea dos pesquisadores e instituições está na tabela 1 , a seguir. 
Tabela 1 - Número de pesquisadores por área e Instituição.

\begin{tabular}{|c|c|c|c|c|c|c|c|c|c|c|c|c|}
\hline \multirow{3}{*}{$\begin{array}{c}\text { Instituiç } \\
\tilde{a} \mathbf{o}\end{array}$} & \multicolumn{10}{|c|}{ Área } & \multirow{3}{*}{$\begin{array}{c}\text { T } \\
\text { ot } \\
\text { al } \\
\mathrm{n}\end{array}$} & \multirow{3}{*}{$\%$} \\
\hline & \multicolumn{2}{|c|}{$\begin{array}{c}\text { Enfermag } \\
\text { em }\end{array}$} & \multicolumn{2}{|c|}{ Medicina } & \multicolumn{2}{|c|}{ Farmácia } & \multicolumn{2}{|c|}{$\begin{array}{c}\text { Odontolog } \\
\text { ia }\end{array}$} & \multicolumn{2}{|c|}{$\begin{array}{c}\text { Saúde } \\
\text { Coletiva }\end{array}$} & & \\
\hline & $\mathrm{n}$ & $\%$ & $\mathrm{n}$ & $\%$ & $\mathrm{n}$ & $\%$ & $\mathrm{n}$ & $\%$ & $\mathrm{n}$ & $\%$ & & \\
\hline FIOCRUZ & 0 & 0 & 0 & 0 & 0 & 0 & 0 & 0 & 8 & 50 & 8 & 20,52 \\
\hline UFSC & 1 & 25 & 0 & 0 & 1 & 16,7 & 0 & 0 & 0 & 0 & 2 & 5,11 \\
\hline UFPel & 0 & 0 & 0 & 0 & 0 & 0 & 0 & 0 & 3 & 18,8 & 3 & 7,72 \\
\hline UFMG & 0 & 0 & 8 & 66,7 & 0 & 0 & 0 & 0 & 0 & 0 & 8 & 20,52 \\
\hline UNESP & 0 & 0 & 0 & 0 & 0 & 0 & 1 & 100 & 0 & 0 & 1 & 2,57 \\
\hline UFPR & 0 & 0 & 1 & 8,3 & 0 & 0 & 0 & 0 & 0 & 0 & 1 & 2,57 \\
\hline UFPE & 0 & 0 & 0 & 0 & 2 & 33,3 & 0 & 0 & 0 & 0 & 2 & 5,11 \\
\hline UFRGS & 0 & 0 & 3 & 25 & 3 & 50 & 0 & 0 & 2 & 12,5 & 8 & 20,52 \\
\hline FURG & 1 & 25 & 0 & 0 & 0 & 0 & 0 & 0 & 0 & 0 & 1 & 2,57 \\
\hline UFC & 2 & 50 & 0 & 0 & 0 & 0 & 0 & 0 & 0 & 0 & 2 & 5,11 \\
\hline UnB & 0 & 0 & 0 & 0 & 0 & 0 & 0 & 0 & 1 & 6,25 & 1 & 2,57 \\
\hline UFBA & 0 & 0 & 0 & 0 & 0 & 0 & 0 & 0 & 2 & 12,5 & 2 & 5,11 \\
\hline Total & 4 & & 12 & & 6 & & 1 & & 16 & & & \\
\hline$\%$ & $\begin{array}{l}10, \\
27 \\
\end{array}$ & 100 & $\begin{array}{c}30,7 \\
6 \\
\end{array}$ & 100 & $\begin{array}{c}15,3 \\
8 \\
\end{array}$ & 100 & 2,57 & 100 & $\begin{array}{c}41,0 \\
2 \\
\end{array}$ & 100 & & \\
\hline
\end{tabular}

Fonte: Dados da pesquisa.

A Saúde Coletiva é a área com o maior número de pesquisadores, com 41,02\% (16) do total de 39, todos vinculados a FIOCRUZ. A Medicina conta com 30,76\% (12) do total, sendo a maioria da UFMG, seguida da UFRGS; a Farmácia, conta com 15,38\% (6) do total, sendo três da UFRGS, dois da UFPE e um da UFSC; na Enfermagem são 10,27\% (4) dos pesquisadores, sendo dois da UFC, um da UFSC e um da FURG; na Odontologia, 2,57\% (1) dos pesquisadores, da UNESP.

Esses pesquisadores publicaram um total de 1.296 artigos em 493 periódicos nos três anos analisados, o que representa uma média de 11 artigos por pesquisador por ano. Os periódicos estão distribuídos em entidades editoras de 24 países, conforme tabela 2 , na sequência. 
Tabela 2 - Distribuição dos artigos por periódicos e países.

\begin{tabular}{|c|c|c|c|c|}
\hline País & $\begin{array}{c}\text { Número de } \\
\text { artigos }\end{array}$ & $\mathbf{\%}$ & $\begin{array}{c}\text { Número de } \\
\text { periódicos }\end{array}$ & $\mathbf{\%}$ \\
\hline Alemanha & 34 & 2,63 & 19 & 3,86 \\
\hline Argentina & 11 & 0,84 & 4 & 0,81 \\
\hline Austrália & 3 & 0,23 & 3 & 0,6 \\
\hline Bélgica & 1 & 0,08 & 1 & 0,21 \\
\hline Bósnia & 1 & 0,08 & 1 & 0,21 \\
\hline Brasil & 463 & 35,72 & 66 & 13,3 \\
\hline Canadá & 1 & 0,07 & 1 & 0,21 \\
\hline Chile & 2 & 0,15 & 2 & 0,41 \\
\hline Colômbia & 1 & 0,08 & 1 & 0,21 \\
\hline Croácia & 2 & 0,16 & 1 & 0,21 \\
\hline Cuba & 2 & 0,16 & 1 & 0,21 \\
\hline Dinamarca & 9 & 0,69 & 4 & 0,81 \\
\hline Espanha & 13 & 1,01 & 9 & 1,82 \\
\hline Estados Unidos & 252 & 19,44 & 135 & 27,2 \\
\hline França & 5 & 0,38 & 4 & 0,81 \\
\hline Grécia & 1 & 0,08 & 1 & 0,21 \\
\hline Holanda & 62 & 4,79 & 36 & 7,5 \\
\hline Hungria & 2 & 0,16 & 2 & 0,41 \\
\hline Índia & 7 & 0,54 & 6 & 1,22 \\
\hline Irlanda & 27 & 2,08 & 7 & 1,41 \\
\hline Itália & 5 & 0,38 & 5 & 1,01 \\
\hline Japão & 2 & 0,16 & 2 & 0,41 \\
\hline Reino Unido & 348 & 26,86 & 164 & 33,5 \\
\hline Suíça & 33 & 2,54 & 16 & 3,04 \\
\hline Não localizado & 9 & 0,69 & 2 & 0,41 \\
\hline Total & 1.296 & 100 & 493 & 100 \\
\hline & & & & \\
\hline
\end{tabular}

Fonte: Dados da pesquisa.

Os resultados mostram o Reino Unido com o maior número de periódicos, com 33,5\% (164) do total, seguido pelos Estados Unidos, com 27,2\% (135), e pelo Brasil, com 13,38\% (66). Os periódicos dos outros 21 países não ultrapassam 50 títulos em cada um, sendo que 16 países têm menos do que cinco títulos identificados. Os dados apontam alta concentração de artigos em apenas dois países.

Apesar de o Reino Unido e os Estados Unidos aparecerem como os países com maior número de periódicos, o mesmo não acontece com o número de artigos publicados. Os 66 periódicos brasileiros publicaram 35,72\% (463) dos artigos de um total de 1.296. O Reino Unido publicou 26,86\% (348) e os Estados Unidos, 19,44\% (252). 
A publicação de artigos em periódicos estrangeiros mostra a internacionalização da área. O termo "internacional" tem sido utilizado há muitos anos entre os membros da comunidade científica como sinônimo de qualidade. Na maior parte dos países, as publicações internacionais são muito mais importantes do que as nacionais (BUELA-CASAL; ZYCH e et al., 2007). Muitos dos artigos são publicados em periódicos que pertencem a grandes editoras comerciais, principalmente, americanas, inglesas e holandesas. As entidades editoras dos periódicos estão descritas na Tabela 3 e são apresentadas por países e por tipo de instituição publicadora. Nas palavras de Mueller (2011), o termo "entidade editora" é utilizado para designar a função do publicador ou publisher.

Tabela 3 - Entidades Editoras dos periódicos.

\begin{tabular}{|c|c|c|c|c|c|c|c|c|c|c|c|c|}
\hline \multirow[b]{2}{*}{ País } & \multirow{2}{*}{$\begin{array}{c}\text { Asso } \\
\text { ciaçã } \\
\text { o }\end{array}$} & \multirow{2}{*}{$\begin{array}{c}\text { Univ } \\
\text { ersid } \\
\text { ade }\end{array}$} & \multicolumn{6}{|c|}{ Editoras Comerciais } & \multirow[b]{2}{*}{$\begin{array}{c}\text { Total } \\
\text { Editora } \\
\text { s } \\
\text { Comerc } \\
\text { iais }\end{array}$} & \multirow{2}{*}{$\begin{array}{c}\text { Outr } \\
\text { os }\end{array}$} & \multirow{2}{*}{$\begin{array}{c}\text { Tota } \\
1\end{array}$} & \multirow[b]{2}{*}{$\%$} \\
\hline & & & $\begin{array}{l}\text { Else } \\
\text { vier }\end{array}$ & $\begin{array}{c}\text { Sprin } \\
\text { ger }\end{array}$ & Wiley & $\begin{array}{l}\text { Natu } \\
\text { re }\end{array}$ & $\begin{array}{c}\text { Bio } \\
\text { Me } \\
\text { d }\end{array}$ & \begin{tabular}{|c|} 
Outras \\
Editor \\
as
\end{tabular} & & & & \\
\hline Alemanha & 0 & 0 & 4 & 13 & 0 & 0 & 0 & 1 & 18 & 1 & 19 & 3,8 \\
\hline Argentina & 1 & 2 & 0 & 0 & 0 & 0 & 0 & 0 & 0 & 1 & 4 & 0,8 \\
\hline Austrália & 0 & 0 & 0 & 0 & 2 & 0 & 0 & 0 & 2 & 1 & 3 & 0,6 \\
\hline Bélgica & 1 & 0 & 0 & 0 & 0 & 0 & 0 & 0 & 0 & 0 & 1 & 0,2 \\
\hline Bósnia & 1 & 0 & 0 & 0 & 0 & 0 & 0 & 0 & 0 & 0 & 1 & 0,2 \\
\hline Brasil & 32 & 27 & 0 & 0 & 0 & 0 & 1 & 1 & 2 & 5 & 66 & 13,5 \\
\hline Canadá & 0 & 0 & 0 & 0 & 0 & 0 & 0 & 1 & 1 & 0 & 1 & 0,2 \\
\hline Chile & 0 & 2 & 0 & 0 & 0 & 0 & 0 & 0 & 0 & 0 & 2 & 0,4 \\
\hline Colômbia & 0 & 1 & 0 & 0 & 0 & 0 & 0 & 0 & 0 & 0 & 1 & 0,2 \\
\hline Croácia & 1 & 0 & 0 & 0 & 0 & 0 & 0 & 0 & 0 & 0 & 1 & 0,2 \\
\hline Cuba & 1 & 0 & 0 & 0 & 0 & 0 & 0 & 0 & 0 & 0 & 1 & 0,2 \\
\hline Dinamarca & 0 & 0 & 0 & 0 & 4 & 0 & 0 & 0 & 4 & 0 & 4 & 0,8 \\
\hline Espanha & 2 & 3 & 3 & 0 & 0 & 0 & 0 & 0 & 3 & 1 & 9 & 1,8 \\
\hline $\begin{array}{c}\text { Estados } \\
\text { Unidos }\end{array}$ & 27 & 5 & 29 & 13 & 16 & 0 & 1 & 35 & 94 & 9 & 135 & 27,5 \\
\hline França & 1 & 0 & 3 & 0 & 0 & 0 & 0 & 0 & 3 & 0 & 4 & 0,8 \\
\hline Grécia & 0 & 0 & 0 & 0 & 0 & 0 & 0 & 1 & 1 & 0 & 1 & 0,2 \\
\hline Holanda & 0 & 0 & 27 & 2 & 0 & 0 & 0 & 3 & 32 & 4 & 36 & 7,3 \\
\hline Hungria & 0 & 0 & 0 & 1 & 0 & 0 & 0 & 1 & 2 & 0 & 2 & 0,4 \\
\hline Índia & 3 & 0 & 1 & 2 & 0 & 0 & 0 & 0 & 3 & 0 & 6 & 1,2 \\
\hline Irlanda & 0 & 0 & 7 & 0 & 0 & 0 & 0 & 0 & 7 & 0 & 7 & 1,4 \\
\hline Itália & 2 & 0 & 0 & 1 & 0 & 0 & 0 & 2 & 3 & 0 & 5 & 1,0 \\
\hline Japão & 1 & 1 & 0 & 0 & 0 & 0 & 0 & 0 & 0 & 0 & 2 & 0,4 \\
\hline $\begin{array}{l}\text { Reino } \\
\text { Unido }\end{array}$ & 11 & 18 & 35 & 6 & 38 & 10 & 30 & 9 & 128 & 7 & 164 & 33,5 \\
\hline Suíça & 4 & 0 & 1 & 2 & 0 & 0 & 0 & 6 & 9 & 3 & 16 & 3,0 \\
\hline N.I & 0 & 0 & 0 & 1 & 0 & 0 & 0 & 1 & 2 & 0 & 2 & 0,4 \\
\hline Total & 88 & 59 & 110 & 41 & 60 & 10 & 32 & 61 & 314 & 32 & 493 & \\
\hline$\%$ & 17,8 & 11,9 & 22,3 & 8,3 & 12,2 & 2,02 & 6,5 & 12,3 & 63,7 & 6,4 & & \\
\hline
\end{tabular}

Fonte: Dados da pesquisa. 
As entidades publicadoras dos periódicos (apresentadas na tabela 3) mostram que as editoras comerciais juntas detêm um total de 63,7\% (314) dos 493 periódicos identificados na pesquisa. A Elsevier aparece com 22,3\% (110) do total, a Wiley, com 12,1\% (60), a Springer, com 8,3\% (41), a Biomed, 6,9\% (32) e a Nature com 2,0\% (10). Outras editoras aparecem com 12,37\% (61) e publicaram até cinco artigos no período analisado. O Reino Unido é o país em que as editoras comerciais aparecem com o maior número de periódicos em que os pesquisadores publicaram, com 128 títulos. Em seguida vêm os Estados Unidos com 94, o Brasil com 66 e a Holanda com 32.

As associações são publicadoras de $17,8 \%$ (88) dos periódicos e as universidades, 11,9\% (59), que, somados, representam 29\% (147) da amostra. No Brasil, as associações publicam 32 periódicos, e as universidades 27, que somadas representam um percentual muito maior $(89 \%)$ do que nos outros países (Estados Unidos com 24\% e Reino Unido com 18\%), dominados pelas editoras comerciais.

Na seção "Outras editoras"1 estão as que aparecem com menos de cinco periódicos e são responsáveis pela publicação de 12,3\% (61) dos títulos. Já na seção "Outros" estão as entidades editoras não identificadas e os Institutos de Pesquisa, Fundações e Organizações, que detêm 6,4\% (32) na publicação dos periódicos.

A pesquisa de Rodrigues e Abadal (2014) também foi realizada com periódicos dos principais países ibero-americanos, num total de 549. No Brasil, foram pesquisados 249 periódicos e, na Espanha, 300. No Brasil, as universidades se destacam com 47\% (118), as associações com 40\% (100) e governos com 3,2\% (8). As editoras comerciais com $0,8 \%$ (2), institutos, 4,8\% (12), outros, $2 \%$ (5). Os autores comentam que as Universidades e Associações são claramente as principais responsáveis pelas revistas, representando um total de $68,8 \%$ (218).

No Brasil, segundo Packer (2011, p. 55), os periódicos “[...] são publicados, em sua maioria, por instituições públicas sem fins lucrativos [...]”. O autor também comenta que, tomando por base a coleção SCIELO, os periódicos brasileiros são publicados por editoras que se dividem em dois grupos: editoras 
de origem institucional (universidades e institutos de pesquisa) e as editoras coordenadas por sociedades científicas ou associações profissionais.

Quanto à forma de acesso ao conteúdo dos periódicos, a opção foi classificar em quatro tipos: a) aberto; b) aberto comercial (no qual todos os artigos estão em acesso aberto); c) subscrição e d) subscrição com opção autor paga.

Tabela 4 - Tipo de acesso aos periódicos.

\begin{tabular}{|c|c|c|c|c|c|c|c|c|c|c|}
\hline \multirow[t]{2}{*}{ País } & \multicolumn{2}{|c|}{ Aberto } & \multicolumn{2}{|c|}{$\begin{array}{c}\text { Aberto } \\
\text { Comercial }\end{array}$} & \multicolumn{2}{|c|}{ Subscrição } & \multicolumn{2}{|c|}{$\begin{array}{c}\text { Subscrição } \\
\text { com opção } \\
\text { de autor } \\
\text { paga }\end{array}$} & \multirow{2}{*}{$\begin{array}{c}\text { Total } \\
\mathbf{n}^{\mathbf{0}} \\
\end{array}$} & \multirow{2}{*}{$\begin{array}{l}\% \\
\% \\
\end{array}$} \\
\hline & $n^{0}$ & $\%$ & $\mathrm{n}^{\mathbf{0}}$ & $\%$ & $\mathrm{n}^{\mathbf{0}}$ & $\%$ & $\mathbf{n}^{\mathbf{o}}$ & $\%$ & & \\
\hline Alemanha & 0 & 0 & 0 & 0 & 12 & 6,7 & 7 & 5,02 & 19 & 3,9 \\
\hline Argentina & 2 & 1,73 & 0 & 0 & 2 & 1,12 & 0 & 0 & 4 & 0,8 \\
\hline Austrália & 1 & 0,86 & 0 & 0 & 2 & 1,12 & 0 & 0 & 3 & 0,6 \\
\hline Bélgica & 0 & 0 & 0 & 0 & 1 & 0,56 & 0 & 0 & 1 & 0,2 \\
\hline Bósnia & 1 & 0,86 & 0 & 0 & 0 & 0 & 0 & 0 & 1 & 0,2 \\
\hline Brasil & 65 & 56,90 & 1 & 1,65 & 0 & 0 & 0 & 0 & 66 & 13,7 \\
\hline Canadá & 0 & 0 & 0 & 0 & 1 & 0,56 & 0 & 0 & 1 & 0,2 \\
\hline Chile & 2 & 1,73 & 0 & 0 & 0 & 0 & 0 & 0 & 2 & 0,4 \\
\hline Colômbia & 1 & 0,86 & 0 & 0 & 0 & 0 & 0 & 0 & 1 & 0,2 \\
\hline Croácia & 0 & 0 & 1 & 1,65 & 0 & 0 & 0 & 0 & 1 & 0,2 \\
\hline Cuba & 1 & 0,86 & 0 & 0 & 0 & 0 & 0 & 0 & 1 & 0,2 \\
\hline Dinamarca & 0 & 0 & 0 & 0 & 3 & 1,68 & 1 & 0,71 & 4 & 0,8 \\
\hline Espanha & 3 & 2,58 & 0 & 0 & 5 & 2,8 & 1 & 0,71 & 9 & 1,8 \\
\hline Estados Unidos & 18 & 15,51 & 6 & 10,3 & 59 & 32,95 & 52 & 37,41 & 135 & 27,9 \\
\hline França & 0 & 0 & 0 & 0 & 3 & 1,67 & 1 & 0,71 & 4 & 0,8 \\
\hline Grécia & 0 & 0 & 0 & 0 & 0 & 0 & 1 & 0,71 & 1 & 0,2 \\
\hline Holanda & 1 & 0,86 & 1 & 1,65 & 7 & 3,91 & 27 & 19,41 & 36 & 7,3 \\
\hline Hungria & 1 & 0,86 & 0 & 0 & 1 & 0,56 & 0 & 0 & 2 & 0,4 \\
\hline Índia & 2 & 1,73 & 0 & 0 & 1 & 0,56 & 3 & 2,15 & 6 & 1,2 \\
\hline Irlanda & 0 & 0 & 0 & 0 & 4 & 2,24 & 3 & 2,15 & 7 & 1,4 \\
\hline Itália & 1 & 0,86 & 1 & 1,65 & 3 & 1,67 & 0 & 0 & 5 & 1.0 \\
\hline Japão & 0 & 0 & 0 & 0 & 2 & 1,12 & 0 & 0 & 2 & 0,4 \\
\hline Reino Unido & 11 & 9,48 & 48 & 83,01 & 69 & 38,54 & 36 & 26,0 & 164 & 34 \\
\hline Suíça & 5 & 4,32 & 0 & 0 & 4 & 2,24 & 7 & 5,02 & 16 & 3,2 \\
\hline Não localizado & 0 & 0 & 0 & 0 & 0 & 0 & 0 & 0 & 2 & 0,4 \\
\hline Total & 115 & \multirow{2}{*}{100} & 58 & \multirow{2}{*}{100} & 179 & \multirow{2}{*}{100} & 139 & \multirow{2}{*}{100} & \multirow[t]{2}{*}{493} & \\
\hline$\%$ & 23,30 & & 11,70 & & 36,0 & & 29 & & & \\
\hline
\end{tabular}

Fonte: Dados da pesquisa.

Observa-se, na tabela acima, que o acesso por subscrição e o acesso por subscrição com opção autor paga somados aparecem em número bem significativo entre os 493 periódicos, representando 65,1\% (318). O acesso 
aberto aparece com 23,3\% (115), e o acesso aberto comercial, em apenas 11,7\% (58) dos títulos.

Na divisão por países, o acesso aberto é mais frequente nos periódicos brasileiros, com 56,90\% (65) de um total de 115 títulos com esta opção, os Estados Unidos aparecem com 15,51\% (18) e o Reino Unido com 9,48\% (11).

O acesso por subscrição é a alternativa escolhida por 179 periódicos. Ainda na tabela 4, cabe destacar Reino Unido, com 38,54\% (69), e Estados Unidos, com 32,95\% (59), nesta opção. Os demais países somam juntos 11,73\% (21) nesse tipo de acesso. O Brasil não possui nenhum periódico em acesso por subscrição na amostra desta pesquisa.

Os Estados Unidos são o país em que o acesso por subscrição com opção autor paga é mais frequente 37,41\% (52). O Reino Unido aparece com 25,9\% (36), a Holanda com 19,43\% (27). Os demais países, conforme tabela 4, apresentam de 0 a 7 periódicos no acesso por subscrição. De um total de 493 periódicos, 11,70\% (58) estão em acesso aberto comercial, sendo que o Reino Unido dispara com o índice maior 84, $22 \%$ (48), os Estados Unidos com 10,53\% (6), o Brasil, a Croácia, a Holanda e a Itália aparecem apenas com um título cada um.

Três países se destacaram na pesquisa: Estados Unidos e Reino Unido no acesso por subscrição e subscrição com opção autor paga e o Brasil no acesso aberto. É possível inferir que os "chamados países centrais" da ciência se concentram em apenas dois nesta área (GUÉDON, 2010).

Do total de 1.296 artigos publicados, os periódicos brasileiros contribuem com 493 artigos (tabela 2) dos 513 publicados em acesso aberto. O acesso por subscrição aparece com $27 \%$ (350) e o com subscrição com opção autor paga, com 21,53\% (279) dos periódicos, todos de editoras comerciais. O acesso aberto comercial aparece com 11,88\% (154) dos artigos publicados. 
Tabela 5 - Tipos de acesso aos artigos.

Fonte: Dados da pesquisa.

\begin{tabular}{|c|c|c|c|c|c|c|c|c|c|c|}
\hline \multirow[t]{2}{*}{ Instituição } & \multicolumn{2}{|c|}{ Aberto } & \multicolumn{2}{|c|}{$\begin{array}{c}\text { Aberto } \\
\text { Comercial }\end{array}$} & \multicolumn{2}{|c|}{ Subscrição } & \multicolumn{2}{|c|}{$\begin{array}{l}\text { Subscrição } \\
\text { com opção } \\
\text { Autor Paga }\end{array}$} & \multicolumn{2}{|c|}{ Total } \\
\hline & $\mathbf{n}$ & $\%$ & $\mathbf{n}$ & $\%$ & $\mathbf{n}$ & $\%$ & $\mathbf{n}$ & $\%$ & $\mathbf{n}$ & $\%$ \\
\hline FIOCRUZ & 129 & 25,15 & 28 & 18,19 & 64 & 18,29 & 25 & 8,97 & 246 & 18,99 \\
\hline UFPel & 58 & 11,3 & 37 & 24,02 & 53 & 15,14 & 33 & 11,82 & 181 & 13,97 \\
\hline UFRGS & 58 & 11,3 & 42 & 27,28 & 84 & 24 & 69 & 24,73 & 253 & 19,53 \\
\hline UFBA & 24 & 4,68 & 11 & 7,14 & 23 & 6,58 & 7 & 2,5 & 65 & 5,01 \\
\hline UFMG & 61 & 11,9 & 27 & 17,53 & 45 & 12,85 & 75 & 26,89 & 208 & 16,04 \\
\hline UFSC & 53 & 10,33 & 0 & 0 & 11 & 3,14 & 0 & 0 & 64 & 4,94 \\
\hline FURG & 41 & 7,99 & 0 & 0 & 0 & 0 & 0 & 0 & 41 & 3,17 \\
\hline UFC & 59 & 11,5 & 0 & 0 & 7 & 2 & 3 & 1,08 & 69 & 5,32 \\
\hline UFPR & 23 & 4,48 & 0 & 0 & 7 & 2 & 15 & 5,37 & 45 & 3,47 \\
\hline UFPE & 7 & 1,37 & 4 & 2,6 & 17 & 4,86 & 23 & 8,25 & 51 & 3,94 \\
\hline UNESP & 0 & 0 & 3 & 1,94 & 21 & 6 & 24 & 8,6 & 48 & 3,7 \\
\hline UnB & 0 & 0 & 2 & 1,3 & 18 & 5,14 & 5 & 1,79 & 25 & 1,92 \\
\hline Total & 513 & 100 & 154 & 100 & 350 & 100 & 279 & 100 & 1.296 & \\
\hline$\%$ & 39,59 & & 11,88 & & 27,00 & & 21,53 & & & 100 \\
\hline
\end{tabular}

A análise da distribuição dos artigos no Brasil é mostrada na Tabela 5. Percebe-se que uma grande parte, 39,59\% (513), encontra-se em acesso aberto, a maioria em títulos brasileiros. Na divisão por instituição, o tipo de acesso por subscrição aparece com mais frequência na UFRGS, com 24\% (84), na FIOCRUZ, com 18,29\% (64), na UFPel, com 15,14\% (53), na UFMG, com $12,85 \%$ (45) e na UFBA, com 6,58\% (23). UFSC, UFC, UFPR, UFPE, UNESP e UnB aparecem juntas com 6,25\% (81) dos artigos.

É possivel identificar também que, de um total de 1.296 artigos, quase a metade (629) está em acesso por subscrição, demostrando que nem todos os artigos financiados com recursos públicos estão disponíveis para acesso, indo no sentido contrário ao que estabelece a Declaração de Berlim (2003, p. 1): “[...] o conhecimento estará incompleto se a informação não for tornada rapidamente acessível e em larga escala à sociedade [...]”.

Para apontar o acervo disponível nos repositórios institucionais, buscouse, nos repositórios de cada instituição, o número de publicações de cada autor. Verificou-se uma baixa adesão dos pesquisadores (Tabela 6). São 153 artigos divididos em oito instituições. Nos repositórios de quatro instituições não foi localizado nenhum artigo dos pesquisadores que fazem parte da amostra. 
Tabela 6 - Número de artigos nos repositórios por instituição e acesso.

\begin{tabular}{|c|c|c|c|c|c|c|c|c|}
\hline Instituição & Aberto & $\begin{array}{c}\text { Aberto } \\
\text { Pago }\end{array}$ & Subscrição & $\begin{array}{c}\text { Subscrição } \\
\text { com opção } \\
\text { autor paga }\end{array}$ & $\begin{array}{c}\text { Embargo } \\
\text { Vencido }\end{array}$ & N I & Total & $\%$ \\
\hline FIOCRUZ & 38 & 5 & 6 & 10 & 1 & 2 & 68 & 44,5 \\
\hline FURG & 8 & 0 & 0 & 0 & 0 & 0 & 8 & 5,22 \\
\hline UFBA & 7 & 0 & 11 & 5 & 3 & 0 & 23 & 15,02 \\
\hline UFC & 9 & 0 & 0 & 0 & 0 & 0 & 9 & 5,87 \\
\hline UFPel & 1 & 0 & 0 & 0 & 0 & 0 & 1 & 0,64 \\
\hline UFRGS & 27 & 3 & 3 & 0 & 2 & 0 & 35 & 22,9 \\
\hline UnB & 4 & 0 & 0 & 1 & 0 & 0 & 5 & 3,25 \\
\hline UNESP & 4 & 0 & 0 & 0 & 0 & 0 & 4 & 2,6 \\
\hline Total & 98 & 8 & 20 & 16 & 6 & 2 & 153 & \multicolumn{2}{|c|}{100} \\
\hline$\%$ & 63,4 & 5,23 & 12,42 & 10,46 & 3,93 & 1,3 & \multicolumn{2}{|c|}{} \\
\hline
\end{tabular}

Fonte: Dados da pesquisa.

A FIOCRUZ é a instituição com maior número de artigos depositados em repositório (68). A UFRGS disponibiliza 35 dos seus artigos, a UFBA, 23, e as demais instituições publicaram 27 em repositórios, o que demonstra a necessidade de políticas de depósito obrigatório no Brasil.

Quanto ao tipo de acesso aos artigos depositados nos repositórios, 98 estão em acesso aberto, 20 estão em acesso por subscrição, 16 estão em acesso por subscrição com opção autor paga, e seis estão com embargo vencido. Os artigos que aparecem com embargo vencido são aqueles que estavam em acesso por subscrição e o período de embargo venceu, tornando-os, assim, de acesso aberto. Segundo Almeida (2014), o período de embargo pode variar de seis a 36 meses, após esse período, o editor deve disponibilizar o conteúdo. De um total de 1.296 artigos, 629 estão em periódicos cujo acesso é por subscrição ou subscrição com opção autor paga. Destes, apenas 36, que representam $6 \%$, estão nos repositórios, confirmando os resultados de Miguel, Chinchilla-Rodríguez e Moya-Anegón (2011) sobre a fragilidade dos repositórios na América Latina.

Chalhub (2010, p. 7) comenta que a publicação em periódicos com avaliação por pares, de acesso livre ou não, não é condição suficiente para que os resultados de pesquisas tenham seu impacto maximizado. Por essa razão, é crescente o número de universidades que disponibilizam trabalhos em repositórios institucionais. Os autores ainda comentam que essa prática é fortemente recomendada para aumentar a visibilidade e o impacto das pesquisas. 
Outra constatação é que aproximadamente 36 dos artigos que estão nos repositórios institucionais (Tabela 6) têm acesso por subscrição ou subscrição com opção autor paga. Temos, assim, uma parcela de pesquisa de ponta realizada no Brasil nas mãos das editoras comerciais e com acesso limitado, não só aos brasileiros, mas também a todos os interessados no tema.

\section{Conclusão}

A pesquisa realizada teve, como objetivo geral, investigar o tipo de acesso da produção científica dos principais pesquisadores do país, da área da Saúde, disponível nos repositórios institucionais. Assim, identificou-se 39 pesquisadores e 12 repositórios, que atendiam os requisitos de composição da amostra: ser pesquisador $1 \mathrm{~A}$, no $\mathrm{CNPq}$, e estar vinculado a uma instituição com repositório, registrado no ROAR e OPENDOAR.

O resultado da pesquisa mostra que foram publicados 1.296 artigos em 493 periódicos científicos. Destes, 87\% (427) foram em periódicos internacionais, compatível com o que se espera da elite pesquisadora na área estudada. Nesse levantamento, as entidades editoras que apareceram com maior número de publicações foram as editoras comerciais, com um total de 63,70\% (314). Universidades e associações aparecem como publicadoras de 29\% (147) dos periódicos.

Esses resultados mostraram a influência da área geográfica, pois se observa uma grande frequência de periódicos ingleses e americanos, sendo que as editoras comerciais têm maior influência nessas áreas. As universidades e associações, por sua vez, são a maioria das publicadoras de periódicos em países latino-americanos, conforme estudos de Rodrigues e Abadal (2014).

A influência das editoras comerciais também é identificada no tipo de acesso aos periódicos. $\mathrm{O}$ acesso por subscrição e o acesso por subscrição com opção autor paga são os que aparecem com frequência superior a 60\%. O acesso aberto sem taxas aparece com 23,5\% (115), a maioria no Brasil, e o acesso aberto comercial com $11,4 \%$ (58).

A análise do tipo de acesso aos artigos mostrou um contraste em relação aos tipos de acesso dos periódicos. Constatou-se que a maioria dos artigos (513) 
está em acesso aberto e 463 artigos foram publicados em periódicos brasileiros. Em acesso por subscrição, identificou-se 350 artigos e, em acesso por subscrição com opção autor paga, localizou-se 279 artigos.

No decorrer da pesquisa, observou-se a baixa frequência de artigos nos repositórios das instituições. De acordo com os dados levantados, aproximadamente $11 \%$ do total dos artigos (1.296) estão nos repositórios institucionais e foram publicados em 77 periódicos.

Conclui-se, desse modo, que os pesquisadores 1A, da área da Saúde, possuem um grande número de artigos (1.296) publicados, principalmente em periódicos americanos e ingleses. Os artigos publicados em periódicos brasileiros se encontram em acesso aberto, mas apenas 153 desses artigos, publicados tanto em periódicos internacionais quanto em nacionais, encontramse nos repositórios das instituições pesquisadas, mostrando, assim, que a ciência nacional, mesmo patrocinada com dinheiro público, não está disponível em acesso aberto nos repositórios, não atingindo a sociedade como um todo.

\section{Referências}

ABADAL, E. Acceso abierto a la ciencia. Barcelona: UOC, 2012. Disponível em: <http://eprints.rclis.org/bitstream/10760/16863/1/2012-acceso-abiertoepiuoc-vfinal-autor.pdf> . Acesso em: 2 ago. 2014.

ALMEIDA, E. Portal de Periódicos Capes: diretrizes e políticas futuras Encontro Regional de Bibliotecas Universitárias. Disponível em: <http://pt.slideshare.net/sibi_ufrj/portal-de-peridicos-elenara-2014042>. Acesso em: 2 set. 2014.

ALVES, B. H. Abordagem métricas: análise de produção cientifica de artigos de e de rede de colaboração cientifica dos docentes do programa de pós-graduação em ciência da informação, na linha de pesquisa organização da informação da UNESP/ Marília. Revista de Iniciação Científica da FFC, v. 9, n. 2, p. 104115, 2009. Disponível em: <http://www2.marilia.unesp.br/revistas/index.php/ric/article/viewFile/248/208>. Acesso em: 16 out. 2014.

ALVES, L. Informação e os sistemas de comunicação científica na ciência da informação. DataGramaZero, v. 12, n. 3, jun. 2011. Disponível em: <http://www.dgz.org.br/jun11/Art_04.htm>. Acesso em: 16 out. 2014. 
BUELA-CASAL, G.; ZYCH, I. How measure the internationality of scientific publications. Psicothema, v. 24, n. 3, p. 435-441, 2012. Disponível em: <http://www.psicothema.com/pdf/4036.pdf>. Acesso em: 10 set. 2014.

\section{CAPES - COORDENAÇÃO DE APERFEIÇOAMENTO DE PESSOAL DE} NÍVEL SUPERIOR. Missão. Disponível em:

<http://www.capes.gov.br/historia-e-missao>. Acesso em: 10 set. 2014b.

CAPES - COORDENAÇÃO DE APERFEIÇOAMENTO DE PESSOAL DE NÍVEL SUPERIOR. Plano Nacional de Pós-Graduação (2011-2020).

Disponível em: <http://www.capes.gov.br/plano-nacional-de-pos-graduacao> Acesso em: 20 ago. 2014a

CHALHUB, T. Acesso aberto à informação científica no Brasil: um estudo das universidades públicas do Estado do Rio de Janeiro. Relatório. Brasília: IBICT, 2010. Disponível em: <http://www.ibict.br/capacitacao-e-ensino/pesquisa-emciencia-da-informacao/pos-doutorado/pesquisas-concluidas-1/acesso-aberto-ainformacao-científica-no-brasil-um-estudo-das-universidades-publicas-doestado-do-rio-de-janeiro/Tania_Relatorio.pdz>. Acesso em: 9 ago. 2013.

CNPQ - CONSELHO NACIONAL DE DESENVOLVIMENTO CIENTÍFICO E TECNOLÓGICO, 2013. Disponível em:

<http://www.cnpq.br/web/guest/noticiasviews/journal_content/56_INSTANCE_ a6MO/10157/1196537>. Acesso em: 9 ago. 2014.

COSTA, S. M. S. Filosofia aberta, modelos de negócios e agências de fomento: elementos essenciais a uma discussão sobre o acesso aberto à informação científica. Ciência da Informação, Brasília, v. 35, n. 2, p. 39-50, maio/ago. 2006. Disponível em: <http://www.scielo.br/pdf/ci/v35n2/a05v35n2.pdf>. Acesso em: 23 set. 2014.

DECLARAÇÃO de Berlin. In: CONFERENCE ON OPEN ACCESS TO KNOWLEDGE IN THE SCIENCES AND HUMANITIES, Berlin, 2003. Electronic proceedings... Disponível em: <http://oa.mpg.de/openaccessberlin/berlindeclaration.html>. Acesso em:. Acesso em: 9 ago. 2014.

FREIRE, J. D. CNPq e o acesso aberto à informação científica. 2011. $274 \mathrm{f}$. Tese (Doutorado em Ciência da Informação) - Faculdade de Ciência da Informação, Universidade de Brasília, Brasília, 2011. Disponível em: <http://repositorio.bce.UnB.br/handle/10482/9385>. Acesso em: 3 ago. 2014.

GARVEY, W. D. The librarian's role as a social scientist. In: GARVEY, W. D. Communication: the essence of science facilitating information among librarians, scientists, engineers and students. London: Pergamon, 1979.

GUMIEIRO, K. A. Modelos de negócios para periódicos científicos eletrônicos de acesso aberto. 2009. $171 \mathrm{f}$. Dissertação (Mestrado em Ciência da Informação) - Faculdade de Economia, Administração, Contabilidade e 
Ciência da Informação e Documentação, Universidade de Brasília, Brasília, 2009. Disponível em: <http://repositorio.bce.UnB.br/bitstream/10482/3251/1/Katiucia_dissertacao.pdf >. Acesso em: 19 set. 2014.

GUANAES, P. C. V.; GUIMARÃES, M. C. S. Modelos de gestão de revistas científicas: uma discussão necessária. Perspectivas em Ciência da Informação, Belo Horizonte, v. 17, n. 1, p. 56-73, jan./mar. 2012. Disponível em: <http://portaldeperiodicos.eci.ufmg.br/index.php/pci/article/view/1383>. Acesso em: 15 dez. 2014.

GUÉDON, J. C. Acesso aberto e divisão entre ciência predominante e ciência periférica. In: FERREIRA, S. M. S. P.; TARGINO, M. das G. (Org.).

Acessibilidade e visibilidade de revistas científicas eletrônicas. São Paulo: Senac São Paulo, 2010. p. 21-75.

GRUSZYNSKI, A. C.; GOLIN, C. Periódicos científicos e a visibilidade da ciência na web: estudo de caso na UFRGS. DataGramaZero, v. 8, n. 3, jun. 2007. Disponível em: <http://www.dgz.org.br/jun07/Art_02.htm>. Acesso em: 20 dez. 2014.

HARNAD, S. Entrevista com Stevan Harnad. Encontros Bibli: revista eletrônica de biblioteconomia e ciência da informação, Florianópolis, n. esp, p. 10-15, $1^{\circ}$ sem. 2007. Disponível em:

<http://www.periodicos.ufsc.br/index.php/eb/article/view/647/518>. Acesso em: 7 dez. 2014.

LEITE, F. C. L. Como gerenciar e ampliar a visibilidade da informação científica brasileira: repositórios institucionais de acesso aberto. Brasília: IBICT, out. 2009. Disponível em:

$<$ http://www.ibict.br/anexos_noticias/repositorios.institucionais.F.Leite_atualiza do.pdf $>$. Acesso em: 12 set. 2014.

MEADOWS, A. J. A comunicação científica. Brasília: Briquet de Lemos, 1999.

MELERO, R.; ABAD GARCIA, F. Revistas open access: características, modelos económicos y tendencias. BiD: textos universitaris de biblioteconomia i documentació, Barcelona, n. 20, jun. 2011. Disponível em:

<http://www2.ub.edu/bid/consulta_articulos.php?fichero=20meler2.htm>. Acesso em: 13 dez. 2014.

MIGUEL, S.; CHINCHILLA-RODRÍGUEZ, Z.; MOYA-ANEGÓN, F. de. Open access and Scopus: a new approach to scientific visibility from the stand point of access. Journal of the American Society for Information Science and Technology, v. 62, n. 6, p. 1-16, 2011. Disponível em: $<$ http://eprints.rclis.org/bitstream/10760/16100/1/Miguel_et\%20al_2011_Open $\% 20$ Access\%>. Acesso em: 16 out. 2014. 
MUELLER, S. P. M. O impacto das tecnologias de informação na geração do artigo científico: tópicos para estudo. Ciência da Informação, Brasília, v. 23, n. 3, p. 309-317, set./dez. 1999. Disponível em:

<http://revista.ibict.br/ciinf/index.php/ciinf/article/viewFile/1148/794>. Acesso em: 18 set. 2014.

MUELLER, S. P. M. Produção e financiamento de periódicos científicos de acesso aberto: um estudo da base SciELO. In: POBLACIÓN, D. A.; WITTER, G.P.; RAMOS, L. M. S. V. C. (Org.). Revistas científicas: dos processos tradicionais às perspectivas alternativas de comunicação. São Paulo: Ateliê Editorial, 2011. p. 201-229.

PACKER, A. L. Os periódicos brasileiros e a comunicação da pesquisa nacional. Revista USP, São Paulo, n. 89, p. 26-61, mar./maio 2011. Disponível em: <http://rusp.scielo.br/scielo.php?script=sci_arttext\&pid=S010399892011000200004\&lng=pt\&nrm=iso >. Acesso em: 27 nov. 2014.

PROSSER, D. The next information revolution - how open access repositories and journals will transform scholarly communication. International Journal of Information, v. 26, n. 3, p. 181-186, jun. 2004.

<http://www.sparceurope.org/resources/SPARC\%20-\%20LIBER.doc>. Acesso em: 18 set. 2014.

RODRIGUES, E. et al. RepositóriUM: criação e desenvolvimento do Repositório Institucional da Universidade do Minho. In: CONGRESSO NACIONAL DE BIBLIOTECÁRIOS, ARQUIVISTAS E DOCUMENTALISTAS, 8., 2004, Estoril. Anais... Estoril, PT: [s.n.], 2004. Disponivel: <http://eprints.rclis.org/archive/00007962/fullmetadata.html>. Acesso em: 21 set. 2014.

RODRIGUES, R. S.; ABADAL, E. Scientific journals in Brazil and Spain: alternative publisher models. Journal of the Association for Information Science and Technology, v. 65, n. 10, p. 2145-2151, 2014.

SCIENTIFIC JOURNALS INTERNATIONAL. Dysfunctional practices in the journal publishing system. 2012. Disponível em:

<http://www.scientificjournals.org/open_acccess_movement.htm>. Acesso em: 19 dez. 2014.

SCIELO em Perspectiva. Quanto custa publicar em acesso aberto?, 2013. Disponível em: <http://blog.scielo.org/blog/2013/09/18/quanto-custa-publicarem-acesso-aberto/\#.Uu6nofvGG7>. Acesso em: 14 dez. 2014.

TOMAÉL, M.; SILVA, T. E. Repositórios institucionais: diretrizes para políticas de informação. In: ENCONTRO NACIONAL DE PESQUISA EM CIÊNCIA DA INFORMAÇÃO, 8., 2007, Salvador. Anais... Salvador. ENANCIB; 2007. Disponível em: 
<http://www.enancib.ppgci.ufba.br/artigos/GT5--142.pdf > Acesso em: 25 ago. 2013

\title{
Publications of Brazilian researchers the health area: the issue of access
}

\begin{abstract}
This research analyzes the scientific production of Brazilian researchers in the health area. The methodology is exploratory, descriptive and documentary with predominantly quantitative assessment. The Corpus of research are the articles published by researchers who have CNPq scholarship in the years 2010, 2011, 2012. The survey sample consists of 39 researchers that are linked to institutions in which the repositories are registered in ROAR and OpenDOAR. The results show that researchers have an especially large number of articles published in American and British journals. The articles published in Brazilian journals are open access. Very little (153) of these articles published both in international journals and in Brazilian journals are the repositories of the surveyed institutions.
\end{abstract}

Keywords: Scientific production. Scientific Communication. Researchers. Scientific journals. Institutional Repositories.

Recebido: 20/07/2015

Aceito: 22/02/2016

\footnotetext{
${ }^{1}$ Mary Ann Liebert, Inc. publishers, Lippincott Williams \& Wilkins, Thieme Medical Publishers, BNJ, Karger AG, Landes Bioscience, Giovanni Fioriti Editore, Bentham Science Publishers, Taylor \& Francis Group, Dove Medical Press Ltd, SAGE Publications, Informa Healthcare, Hindawi Publishing Corporation, Wichtig Editore, Spandidos Publications, Thieme Verlagsgruppe, PLoS One.
} 